Ny Riana Solomalala Andriamarosoa Ratsimbazafy ${ }^{1}$ Clémence Dirac Ramohavelo ${ }^{2}$ Jean-Pierre Sorg ${ }^{2}$

Alexandre Buttler ${ }^{3}$

1 École supérieure des sciences
agronomiques
Département des Eaux et Forêts
BP 3044

101 Antananarivo

Madagascar

<rianasolomalala@yahoo.fr>

2 ITES

GFD - Groupe de foresterie

pour le développement

ETHZ

Universitätstrasse 22

8092 Zürich

Suisse

<clemence_dirac@hotmail.com>

<jean-pierre.sorg@env.ethz.ch>

3 École polytechnique fédérale de Lausanne

Laboratoire des systèmes écologiques

Institut fédéral de recherches WSL

Site Lausanne

EPFL ENAC

IIE ECOS

GR B2 417

(Bâtiment GR)

Station 2

1015 Lausanne

Suisse

<alexandre.buttler@epfl.ch>

Tirés à part : C. Dirac Ramohavelo

\section{Optimisation socio-économique et écologique des systèmes d'élevage (Menabe central, Madagascar)}

\section{Résumé}

La forêt dense sèche du Menabe central, côte ouest de Madagascar, disparaît à un taux de $1 \%$ par année. Les pratiques de culture sur brûlis font que la forêt est principalement convertie en terrains agricoles dont certains, une fois abandonnés, sont recyclés en pâturages. En maintenant les anciennes zones boisées en savanes ouvertes, les pâtures participent à la dégradation forestière. S'insérant dans un contexte de conservation de la biodiversité et de respect des besoins des populations locales, cette étude présente : i) les pressions de pâture et ii) un diagnostic de la gestion et de l'économie traditionnelle du petit et du grand élevage. La méthodologie utilisée relève des sciences naturelles (végétation des pâturages, biomasse), de l'agriculture (pression de pâture) et des sciences socio-économiques (enquêtes, questionnaires, affectation de scores). La plupart des pâturages étudiés sont dominés par des espèces pyrophytes et sont en situation de surpâturage durant la saison sèche. Cela conduit à une dégradation de la végétation et des sols, que seule une gestion spatio-temporelle adaptée des pâturages pourrait freiner. Le rôle local du grand élevage est à la fois social et économique, selon l'ethnie des éleveurs. Au Menabe central, un système traditionnel de grand élevage est économiquement rentable uniquement si la commercialisation du lait représente plus de la moitié des revenus totaux; cependant, seulement $7 \%$ des éleveurs vendent parfois du lait. L'intensification du système pastoral passe donc par l'optimisation de la filière laitière. Selon la coutume locale, la vente de produits du petit élevage est plus fréquente que celle des produits du grand élevage. En effet, $73 \%$ des éleveurs vendent au moins un animal par année, et $23 \%$ des ménages éleveurs vendent des œufs. Le développement de cette filière nécessiterait cependant un appui à l'amélioration sanitaire, en particulier celvi des poules.

Mots clés : biodiversité, composition floristique, développement rural, enquête, production agricole.

\section{Abstract}

\section{Socio-economical and ecological optimisation of livestock systems} (Central Menabe, Madagascar)

The dry dense forest of Central Menabe, west coast of Madagascar, is disappearing at a rate of $1 \%$ per year. Slash and burn cultivation practices convert the forest soils to agricultural lands, some of which are, once abandoned, reused as pasture lands. By keeping former forest areas in open savannahs, pastures contribute to forest degradation. Within a context of conservation of biodiversity and improvement of livelihood, this study presents i) the pasture pressures, and ii) a diagnostic of the traditional management and economy of small and big livestock. The methodology comes from natural sciences (pasture land vegetation, biomass), from agricultural sciences (pasture pressures) and from socio-economical sciences (surveys, questionnaires, score assignment). Most of the pasture lands studied are 
dominated by pyrophyte species and overpastured in the dry season. This situation leads to vegetation and soil degradation that can by decreased only by suitable spatial and temporal pasture land management. The local role of big livestock is both social and economical, relating to the breeder's ethnicity. In Central Menabe, a traditional big livestock system is economically profitable only if the milk marketing represents more than the half of the total income; only $7 \%$ of the breeders, however, sometimes sell milk. The pastoral system intensification is thus linked to milk marketpath optimisation. According to local custom, small livestock products participate more frequently in the rural households' budgets as $73 \%$ of the breeders sell at least one animal per year and $23 \%$ sell eggs. Intensifying the small livestock system would however require support for an improvement in chicken sanitary practices.

Key words: agricultural production, biodiversity, floristic composition, rural development, survey.

a forêt dense sèche du Menabe central, côte ouest de Madagascar, disparaît à un taux d'environ $1 \%$ par année [1]. Elle est principalement convertie en terrains agricoles qui, une fois abandonnés, évoluent vers diverses formations secondaires. Certaines formations secondaires de type savane arborée à graminées vivaces héliophiles sont utilisées comme pâturages pour les bovins (figure 1) et les caprins (figure 2) élevés en mode d'élevage extensif et semiintensif. En maintenant les anciennes zones boisées en savanes ouvertes, ces pâtures - indispensables au bétail - participent à la dégradation forestière et empêchent sa

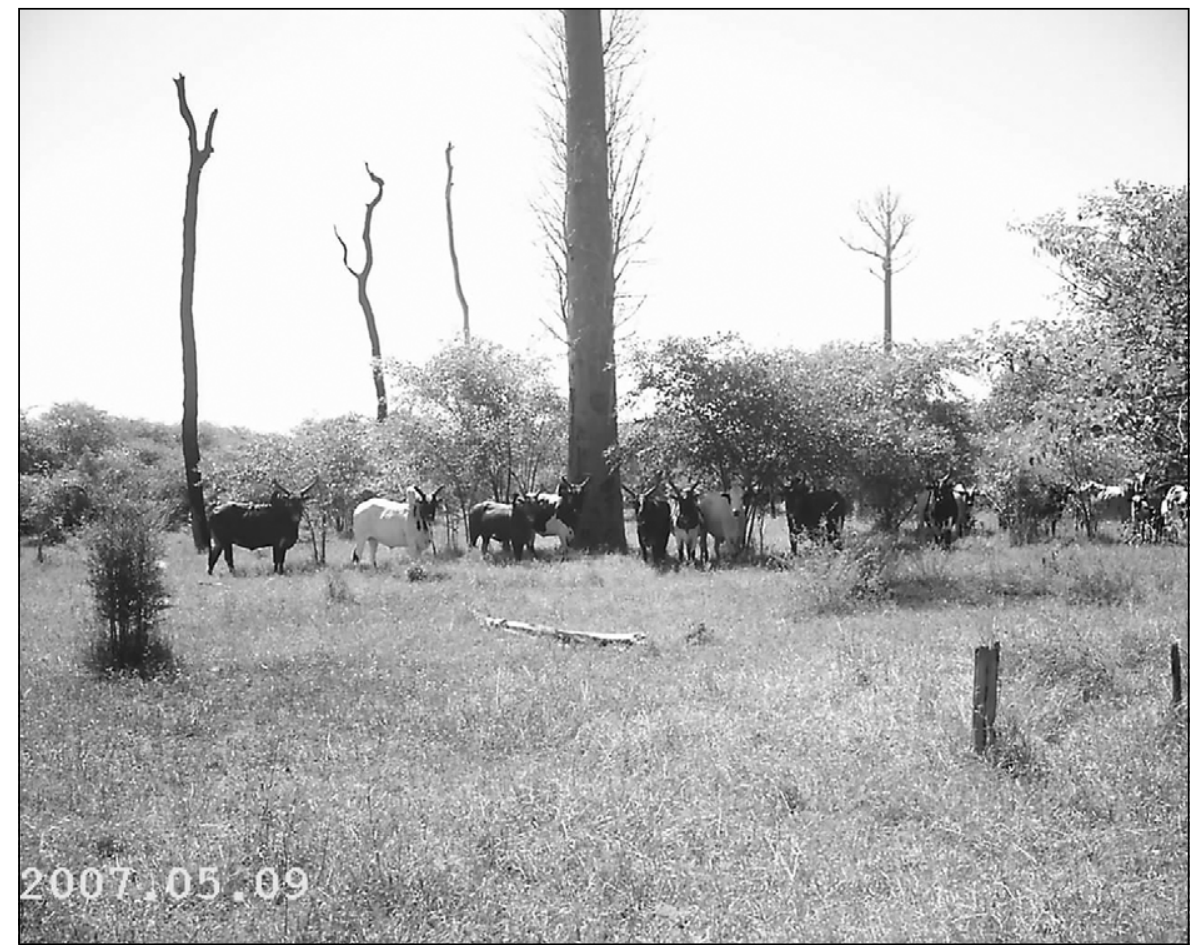

Figure 1. Les zébus semi-sauvages du Menabe central errent dans les zones boisées. Plus le troupeau est grand, plus le niveau social du propriétaire est élevé (cliché : N. R. S. Andriamarosoa Ratsimbazafy).

régénération. En outre, le feu est traditionnellement utilisé pour le renouvellement des pâturages; ces feux sont parfois mal contrôlés, ils se propagent dans les zones boisées et accentuent la déforestation [2]. L'élevage de bovins, qui se diversifie vers l'élevage d'autres animaux (ovins, caprins, porcins et volailles) [3], représente une des principales activités de la région du Menabe.

Afin de diminuer la dégradation forestière dans les pays en développement, les recherches actuelles tendent à optimiser l'utilisation mixte du terroir dans le double but de protéger la biodiversité et d'améliorer les conditions de vie des populations rurales
[4]. Étant donné qu'à Madagascar 68,7\% de la population vit encore en dessous du seuil de pauvreté [5], la présente recherche vise premièrement à évaluer les pressions bovines et caprines dans les zones pâturées, et deuxièmement à effectuer un diagnostic de la gestion traditionnelle du petit et du grand élevage. Dans le but $d$ 'augmenter les revenus des villageois tout en diminuant l'impact de la gestion traditionnelle des pâturages sur la biodiversité, des recommandations sont proposées.

\section{Méthodologie}

\section{Choix des villages d'étude et des sites pâturés}

La région du Menabe est caractérisée par deux saisons bien distinctes. La saison des pluies - très chaude - dure de novembre à mars-avril, et la saison sèche - plus fraiche s'étend de mai à août. Septembre et octobre sont des mois de transition, relativement chauds avec quelques précipitations. Les villages étudiés sont ceux d'Ampataka ( $20^{\circ} 04^{\prime} 28,42^{\prime \prime} \mathrm{S} ; 44^{\circ} 28^{\prime} 41,00^{\prime \prime} \mathrm{E}$ ), d'Ankoraobato $\left(20^{\circ} 09^{\prime} 05,55^{\prime \prime} \mathrm{S}\right.$; $44^{\circ}$ $\left.37^{\prime} 35,41^{\prime \prime} \mathrm{E}\right)$, de Mandroatsy (19० 59'

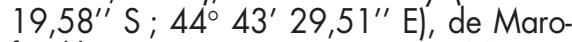
fandilia $\left(20^{\circ} 07^{\prime} 11,07^{\prime \prime} \mathrm{S}\right.$; $44^{\circ} 33^{\prime}$ $\left.23,97^{\prime \prime} \mathrm{E}\right)$, de Kirindy $\left(20^{\circ} 03^{\prime} 12,97^{\prime \prime} \mathrm{S}\right.$; $\left.44^{\circ} 35^{\prime} 19,03^{\prime \prime} \mathrm{E}\right)$ et de Beroboka

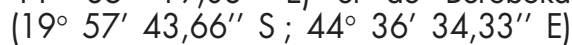
dans le Menabe central. Tous ont une activité d'élevage. Pour l'étude de terrain sur les pressions de pâture, les différents types de pâturages ont été sélectionnés uniquement dans les villages d'Ampataka et d'Ankoraobato, sur la base d'une typologie établie au moyen de la méthode zuricho-montpelliéraine de Braun-Blanquet [6] ; le taux de recouvrement, l'abondance et la dominance des principales espèces ont été estimés dans 60 parcelles (30 par village) mesurant chacune $1 \mathrm{~m}^{2}$. Cela a 
conduit à retenir entre quatre et six sites pâturés par terroir (tableau 1). Les deux terroirs d'Ampataka et d'Ankoraobato sont également ceux qui ont été choisis pour l'étude de la filière du grand élevage.
Estimation de la capacité de charge et de la charge animale

Afin de déduire la pression animale dans les zones pâturées, la capacité de charge

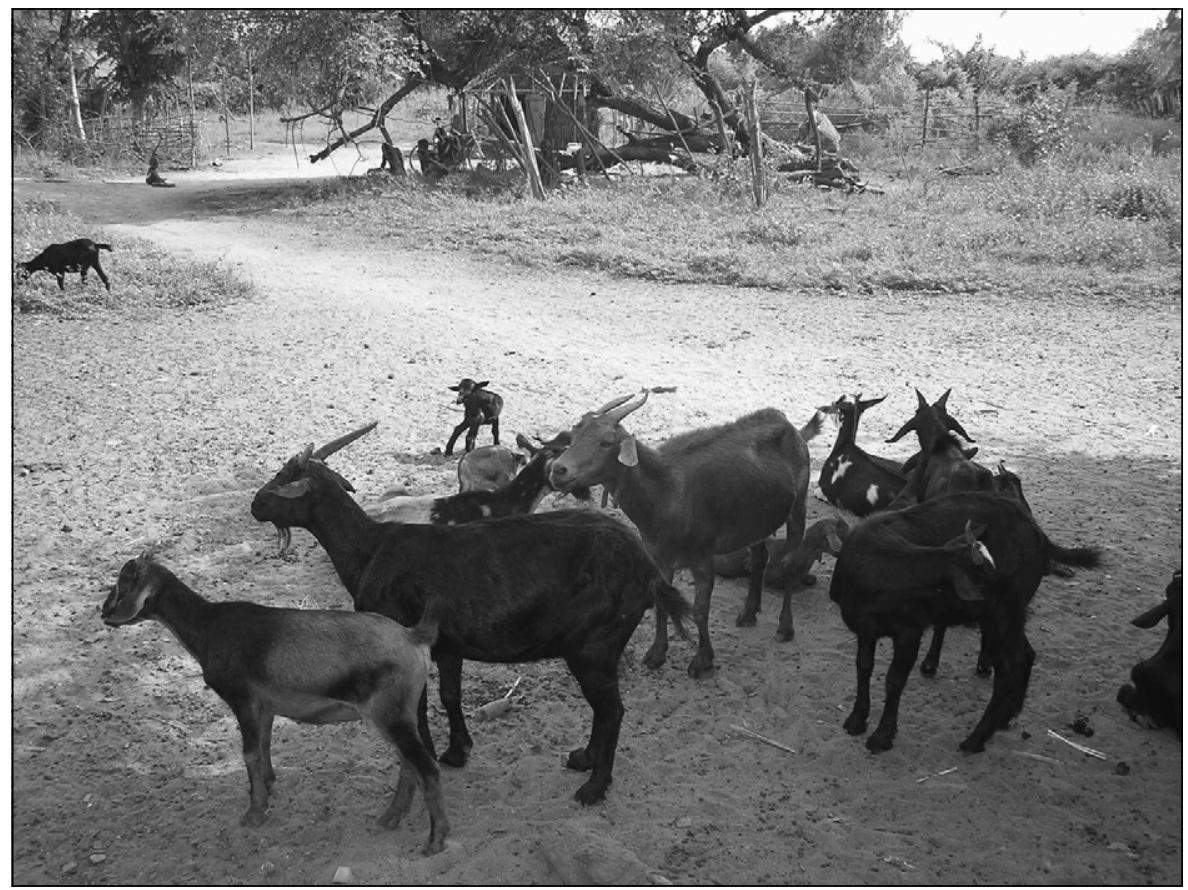

Figure 2. Dans la région du Menabe central, certains troupeaux de chèvres pâturent dans les zones boisées la journée et retournent au village le soir (cliché : L. H. Andriambelo).

Tableau 1. Caractéristiques pastorales des sites étudiés.

\begin{tabular}{|rccllllllllll|}
\hline & AV & BFSP & BFSS & K & S & NSP & NSS & CCSP & CASP & CCSS & CASS & Surpâture \\
\hline \multirow{6}{*}{ AMP } & 1 & 1600 & 800 & 0,6 & 439 & 49 & 243 & 1,27 & 0,11 & 0,31 & 0,55 & Saison sèche \\
& 2 & 3320 & 1660 & 0,6 & 504 & 49 & 275 & 2,63 & 0,10 & 0,65 & 0,55 & Absente \\
& 3 & 3300 & 1650 & 0,6 & 511 & 49 & 275 & 2,62 & 0,10 & 0,65 & 0,54 & Absente \\
& 4 & 1880 & 940 & 0,6 & 1223 & 162 & 243 & 1,49 & 0,13 & 0,37 & 0,20 & Absente \\
& 5 & 2730 & 1365 & 0,6 & 322 & 275 & 243 & 2,17 & 0,85 & 0,54 & 0,75 & Saison sèche \\
& 6 & 1660 & 830 & 0,6 & 690 & 275 & 243 & 1,32 & 0,40 & 0,33 & 0,35 & Saison sèche \\
\hline \multirow{4}{*}{ ANK } & 7 & 2970 & 1485 & 0,7 & 44 & 110 & 183 & 2,75 & 2,50 & 0,68 & 4,16 & Saison sèche \\
& 8 & 1440 & 720 & 0,6 & 430 & 366 & 439 & 1,14 & 0,85 & 0,28 & 1,02 & Saison sèche \\
& 9 & 1890 & 945 & 0,5 & 3707 & 622 & 659 & 1,25 & 0,17 & 0,31 & 0,18 & Absente \\
& 10 & 2330 & 1165 & 0,6 & 454 & 402 & 428 & 1,85 & 0,89 & 0,46 & 0,94 & Saison sèche \\
\hline
\end{tabular}

AMP : Ampataka ; ANK : Ankoraobato ; BFSP : biomasse fourragère en saison des pluies en kilogramme de matière sèche par hectare; $\mathrm{BFSS}$ : biomasse fourragère en saison sèche en kilogramme de matière sèche par hectare, $\mathrm{BFSS}=\mathrm{BFSP} / 2$ (8) ; K : coefficient d'utilisation de la biomasse de l'espèce herbeuse la plus abondante, allant de 0,1 à 0,9 pour les tapis herbacés; $S$ : superficie des sites en hectare; NSP : nombre d'unités bovins tropicaux (UBT) en saison des pluies; NSS : nombre d'unités bovins tropicaux (UBT) en saison sèche ; CCSP : capacité de charge en saison des pluies; CASP : charge animale en saison des pluies; CCSS : capacité de charge en saison sèche ; CASS : charge animale en saison sèche. La surpâture est estimée par comparaison des grandeurs de capacité de charge (CC) et de charge animale (CA).

$\mathrm{AV}$ : association végétale : 1 : Pâturage à Panicum voeltzkowii et Sporobolus festivus ; 2 : Pâturage à Heteropogon contortus, Cynodon dactylon et Panicum voeltzkowii; 3 : Pâturage à Cynodon dactylon et Panicum voeltzkowii ; 4 : Pâturage à Panicum voeltzkowii ; 5 : Pâturage à Heteropogon contortus, Cynodon dactylon, Panicum voeltzkowii et Sporobolus festivus ; 6 : Pâturage à Heteropogon contortus ; 7 : Pâturage à Heteropogon contortus et Hyparrhenia rufa ; 8 : Pâturage à Heteropogon contortus ; 9 : Pâturage à Brachiaria nana ; 10 : Pâturage à Brachiaria nana, Heteropogon contortus, Hyparrhenia rufa et Cynodon dactylon. et la charge animale ont été calculées dans les $10(6+4)$ sites retenus sur les deux terroirs étudiés (Ampataka et Ankoraobato). Les calculs de la capacité de charge et de la charge animale se font suivant les formules $[7,8]$ :

$$
\begin{gathered}
C A=\frac{N}{S} \\
C C=\frac{B F * K}{D U * 6.25}
\end{gathered}
$$

- CC : capacité de charge en UBT (unité bovin tropical par hectare: 1 bovin $=1$ UBT ; 1 caprin $=0.12$ UBT [7]) ;

- $\mathrm{BF}$ : biomasse fourragère en kilogrammes de matière sèche par hectare ;

- K : coefficient d'utilisation de la biomasse allant de 0,1 à 0,9 pour les tapis herbacés

- DU : durée d'utilisation de la biomasse (121 jours en saison humide et 244 jours en saison sèche [9]) ;

- Coefficient 6,25: matière sèche ingérée par 1 UBT .

- CA : charge animale en UBT par hectare ; $-\mathrm{N}$ : nombre d'UBT :

- $S$ : surface du pâturage (site) en hectares.

Si $C C>C A$ : absence de pression

Si $C C<C A$ : présence de pression (surpâturage)

Pour obtenir les valeurs des variables nécessaires à l'estimation de la capacité de charge et de la charge animale, quatre méthodes différentes ont été utilisées.

1. La biomasse fourragère $(B F)$ a été calculée grâce à une analyse quantitative en laboratoire de la phytomasse sèche des espèces dominantes. Dans 60 quadrats de $1 \mathrm{~m}^{2}$ chacun $(30$ quadrats pour chaque terroir), une coupe de la matière verte à $5 \mathrm{~cm}$ du sol a été effectuée; pour chaque espèce, la phytomasse verte et la phytomasse sèche laprès deux séchages dans l'étuve à $70 \pm 1^{\circ} \mathrm{C}$ et à $103 \pm 1^{\circ} \mathrm{C}$ pendant 4 heures) ont été pesées.

2. La superficie pâturée de chaque site $(S)$ a été estimée au moyen de techniques de zonage (relevés GPS) et de données cartographiques.

3. Soixante-sept enquêtes sur les cheptels caprin et bovin et la consultation des cahiers de zébus (livrets qui présentent annuellement le nombre de têtes de bovins par éleveur) ont permis de calculer le nombre d'animaux (N) pâturant dans les différents sites.

4. Des observations directes sur dix troupeaux de bovins aux pâturages ont permis d'estimer le degré de broutage $(\mathrm{K})$ de chaque espèce fourragère selon son appétence et son accessibilité. 


\section{Questionnaires sur la filière "grand élevage »}

Des questionnaires sur la filière "grand élevage » (bovins et caprins) ont été effectués auprès de 80 ménages $(N=26$ dans le village d'Ampataka et $\mathrm{N}=54$ dans celui d'Ankaraobato, ce qui représente la totalité des ménages possédant des troupeaux dans les deux villages). Les questionnaires devaient d'abord permettre de déterminer les rôles traditionnels du système "grand élevage » en quantifiant le pourcentage d'éleveurs de différentes ethnies, le pourcentage de chaque type d'animal acheté et la rentabilité du système " grand élevage ». Afin de connaître la rentabilité du système "grand élevage ", une analyse coût-bénéfice a été effectuée sur la base des données concernant les ventes et les achats d'animaux, les ventes de lait, et les coûts des traitements vétérinaires, du gardiennage et des suppléments alimentaires. La rentabilité du système a été évaluée grâce au calcul de la somme des coûts (valeurs négatives) et des revenus (valeurs positives) engendrés par le système ; si le bilan est positit, le système est économiquement rentable, avec des valeurs négatives il est déficitaire. Pour terminer, une analyse de la filière lait a été effectuée grâce à des données sur le nombre moyen de mois de traite, le pourcentage de lait consommé, le pourcentage d'éleveurs qui vendent du lait et le nombre moyen de litres de lait par traite.

\section{Enquêtes, questionnaires et scoring sur la filière " petit élevage »}

Dans les six villages d'étude, 18 enquêtes qualitatives (à questions ouvertes) ont tout d'abord permis de décrire la gestion traditionnelle du système "petit élevage " (porcins et volailles), et en particulier de faire ressortir les principaux problèmes de la filière.

Ensuite, 288 questionnaires quantitatifs (à questions fermées) avaient pour but d'estimer le pourcentage de ménages possédant au moins un animal de petit élevage, les types d'animaux détenus, le pourcentage de ménages vendant les produits du petit élevage et la difficulté de l'écoulement commercial des produits du petit élevage. 48 ménages ont été sondés dans chaque village. Dans le but de travailler avec un échantillon de population représentatif du village, les 48 ménages enquêtés devaient habiter à différents emplacements géographiques dans le village, car généralement, les maisons des différentes ethnies et des différents lignages se retrouvent groupées. Le nombre de ménages enquêtés dans chaque emplacement géographique était représentatif du nombre de ménages qui y habitait.
Des personnes des deux sexes, de différents âges et de différents niveaux sociaux ont été interviewées.

Afin de définir le rôle socioéconomique du petit élevage dans le système rural, un exercice de scoring - affectation de scores à différents produits - [10] a été effectué en groupe dans chacun des six villages. Dans chaque village, l'exercice a été fait séparément à deux reprises, une fois avec un groupe d'hommes et une fois avec un groupe de femmes. Les groupes se composaient d'une dizaine de personnes pour la plupart précédemment sélectionnées selon leur âge, leur ethnie et leur richesse, le but étant de travailler avec un groupe représentatif du village. Cent jetons ont été distribués également aux participants. Une question était ensuite posée à tout le groupe. Les participants avaient le droit de discuter entre eux pour échanger leurs points de vue, mais chacun devait répondre à la question individuellement en posant un ou plusieurs jetons dans l'une ou plusieurs des cases réponses, selon l'importance accordée à la case réponse. Une case " autre » étant également à disposition. Suivant cette méthode, les villageois ont estimé le pourcentage d'animaux qu'ils élevaient à différentes fins (épargne, ventes, consommations, etc.). La différence statistique entre les pourcentages attribués à l'épargne et aux autres fins des produits du petitélevage a été calculée au moyen du test U de Mann-Whitney. Ce test a été choisi, car d'une part les données récoltées avec les exercices de scoring ne sont pas nombreuses (au total, 12 différents groupes ont participé aux exercices), et d'autre part, les données sont non appariées (la rubrique " autre » $n^{\prime} a$ pas été considérée dans les analyses).

\section{Scoring sur les attentes locales} vis-à-vis des filières "élevage »

Dans le but d'améliorer la gestion des filières "élevage", des exercices de scoring se concentrant sur l'intérêt porté par les éleveurs à des nouvelles pratiques d'élevage plus rentables et plus respectueuses de la biodiversité ont été réalisés, selon la méthode décrite précédemment. Pour chacun des deux élevages, six techniques étaient présentées aux villageois. Les différences statistiques entre les pourcentages attribués aux trois techniques les plus appréciées et ceux attribués aux autres techniques ont été calculées au moyen du test $U$ de Mann-Whitney.

\section{Résultats}

\section{Estimation de la capacité de charge et de la charge animale}

Les pâturages ont l'aspect d'une savane arborée claire soit à palmiers (Medemia nobilis (Hildebr. \& H.Wendl.) Gallerand, Phoenix reclinata Jacq.), soit à espèces ligneuses (Kigelianthe madagascariensis (Baker) Sprague ex H. Perrier, Tamarindus indica L., Ziziphus jujuba Lam.). Ce type de formation résulte de la disparition de la végétation naturelle défrichée pour les cultures ou brûlée pour le renouvellement des pâturages. Ziziphus jujuba et Medemia nobilis offrent des fourrages arborés et arbustifs à base de feuilles, de fruits et de jeunes pousses; les fourrages herbacés sont principalement composés de graminées vivaces, dont les espèces dominantes sont Brachiaria nana Stapf., Cynodon dactylon (L.) Pers., Heteropogon contortus (L.) P. Beauv. ex Roem. \& Schult., Hyparrhenia rufa (Nees) Stapf., Panicum voeltzkowii Mez. et Sporobolus festivus Hochst. ex A. Rich. Les pâturages sélectionnés représentent 10 types d'associations végétales (tableau 1). En période sèche, $60 \%$ des associations végétales $(29 \%$ de la surface de pâture) sont en surpâturage (tableau 1), ce qui empêche la régénération forestière. Globalement, la surface de pâture est dominée par des espèces pyrophytes (telles Brachiaria nana et Panicum voeltzkowii) à faible production fourragère (tableau 2).

La spécificité du terroir d'Ampataka réside sur l'inexistence de bas-fonds, et sur la présence d'une plaine côtière à recouvrement sableux, favorable au développement de Panicum voeltzkowii (tableau 2). Alors que I'association Heteropogon contortus, Cynodon dactylon et Panicum voeltzkowii se trouve dans des zones peu cultivées à proximité du village, Heteropogon contortus devient plus abondant dans les écosystèmes périforestiers. Dans des lieux plus humides (présence de mares temporaires), Panicum voeltzkowii s'associe à Sporobolus festivus.

L'aire pastorale d'Ankoraobato est dominée par une espèce à faible production fourragère (Brachiaria nana), tandis que les espèces produisant une biomasse fourragère supérieure à 3 tonnes par hectare Itelles Cynodon dactylon et Hyparrhenia rufa) occupent $5 \%$ de l'aire pastorale (tableau 2). Brachiaria nana occupe principalement des clairières, des jachères, des forêts de successions secondaires et des forêts dégradées. Sur des sols de bas de pente à une altitude moyenne de $60 \mathrm{~m}$ et une isohyète comprise entre 800 et $1000 \mathrm{~mm}$, elle disparaît progressivement pour laisser place à Heteropogon contortus et à Hyparrhenia rufa; ces sols riches en matières organiques, profonds et humides, sont favorables au développement de l'association Heteropogon contortus et Hyparrhenia rufa. Sur les bords de mares, Brachiaria nana se mêle à Heteropogon contortus, Hyparrhenia rufa et Cynodon dactylon. 
Tableau 2. Production fourragère moyenne (tonne de matière sèche pour un hectare $d^{\prime}$ une espèce dans un milieu donné) et taux de couverture des principales espèces fourragères (pourcentages).

\begin{tabular}{|llcc|}
\hline Terroir & Espèce & $\begin{array}{c}\text { Production fourragère } \\
\text { moyenne }\end{array}$ & $\begin{array}{c}\text { Taux de couverture } \\
\text { (\%) }\end{array}$ \\
\hline Ampataka & Panicum voeltzkowii & $1,90 \pm 0,67$ & 49 \\
& Heteropogon contortus & $2,69 \pm 1,54$ & 30 \\
& Cynodon dactylon & $3,85 \pm 1,60$ & 8 \\
Ankoraobato & Sporobolus festivus & $1,39 \pm 0,17$ & 5 \\
& Brachiaria nana & $1,88 \pm 0,64$ & 81 \\
& Heteropogon contortus & $1,88 \pm 1,00$ & 14 \\
& Cynodon dactylon & $3,27 \pm 0,82$ & 4 \\
& Hyparrhenia rufa & $3,33 \pm 1,12$ & 1 \\
\hline
\end{tabular}

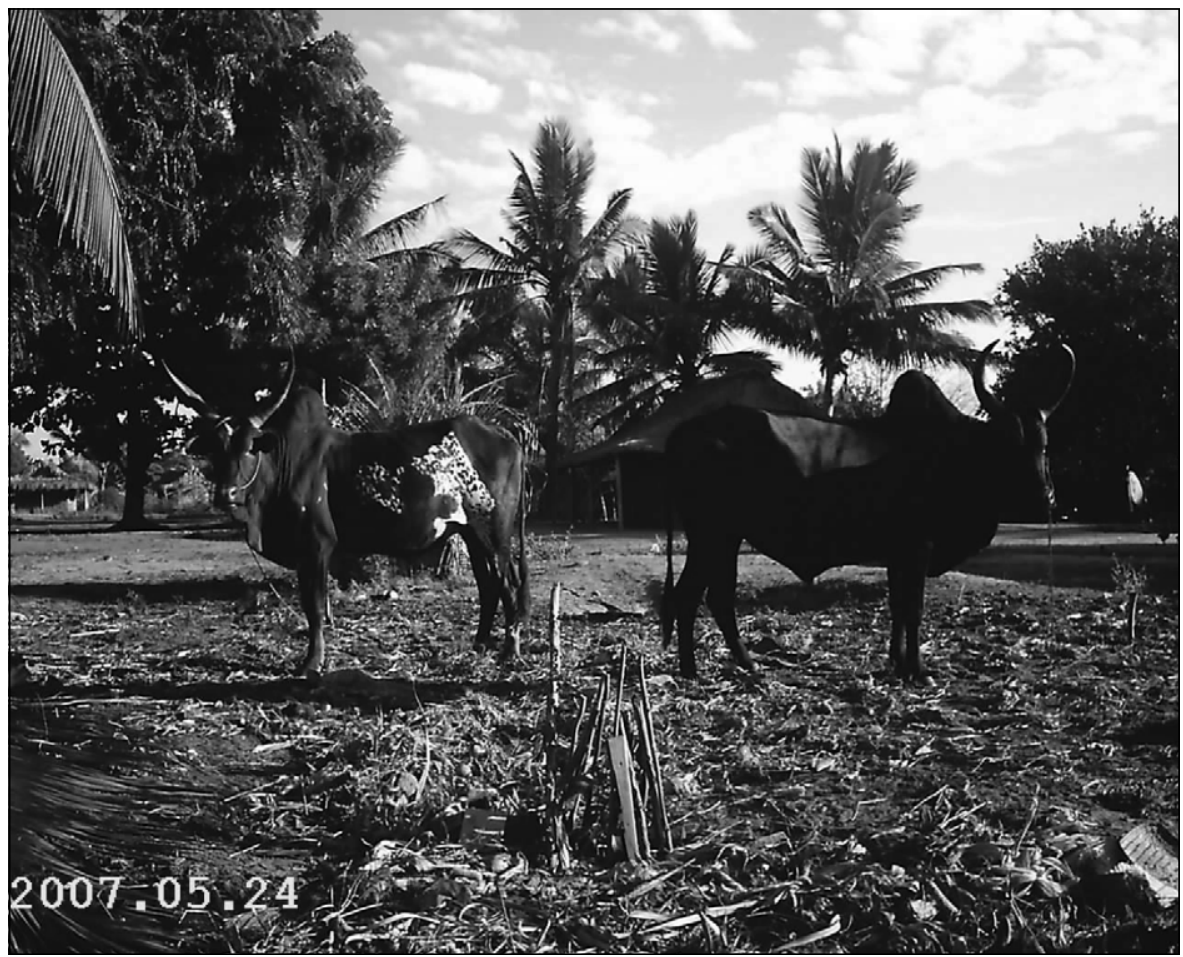

Figure 3. Les zébus de trait sont généralement gardés au village et utilisés comme force de travail (cliché : N. R. S. Andriamarosoa Ratsimbazafy).

\section{Questionnaires, enquêtes et scoring}

- Le « grand élevage»

Les analyses des questionnaires concernant la filière "grand élevage » montrent que dans les villages d'Ampataka et d'Ankoraobato, les éleveurs appartiennent principalement à trois ethnies: à Ampataka, $100 \%$ des éleveurs sont Sakalava (ethnie indigène), et à Ankoraobato $60 \%$ sont Antesaka et $40 \%$ sont Antandroy (ethnies immigrantes). Ensuite, elles confirment que le rôle traditionnel du grand élevage est social et économique. Le rôle social consiste à posséder un grand troupeau élevage), les produits dérivés de l'élevage bovin sont principalement le lait et la viande. Mises à part les fêtes traditionnelles, les abattages de caprins ou de bovins au village et la consommation ou la vente de la viande sont rares, sauf si un animal est très malade. En moyenne, les vaches lqui représentent $16 \%$ des animaux du grand élevage) sont traites pendant $3 \pm 1$ mois (de janvier à mars) avec une production moyenne de $2 \pm 1$ litres par traite. II s'avère que $99 \%$ du lait est destiné à la consommation du ménage, et qu'uniquement $7 \%$ des éleveurs vendent parfois du lait. La consommation de lait est surtout la conséquence des difficultés liées à son stockage.

Pour les éleveurs Sakalava d'Ampataka, le rôle social est primordial. Dans ce village, $100 \%$ des achats d'animaux concernent des mâles qui seront utiles plus tard pour les fêtes traditionnelles. De plus, la somme des coûts et des revenus du système " grand élevage bovin et caprin » d'Ampataka est négative, ce qui signifie que ce système $\mathrm{n}^{\prime}$ est pas économiquement rentable (tableau 3). Les éleveurs immigrants $d$ 'Ankoraobato mènent une conduite d'élevage reposant sur l'accumulation de têtes pour la capitalisation de revenus, ce qui est économiquement rentable (la somme des coûts et des revenus du système " grand élevage bovin et caprin » d'Ankoraobato est positive; tableau 3). En effet, $55 \%$ des achats annuels d'animaux portent sur des femelles, donc de futures reproductrices ou de futures productrices de lait. Contrairement à Ampataka, la commercialisation du lait concerne plus de la moitié des revenus du système " grand élevage » d'Ankoraobato (tableau 3).

Le scoring sur les attentes locales vis-à-vis de la filière "grand élevage » montre que les trois techniques qui ont, statistiquement (U : $p<0,01)$, le plus intéressé les éleveurs sont l'établissement de lieux de pâture fixes, le parcage des animaux et l'abolition des feux de pâturage (figure 4).

- Le " petit élevage»

Les questionnaires et les enquêtes sur la filière "petit élevage » montrent qu'au Menabe central, le petit élevage est une activité fréquente. En effet, $75 \%$ des ménages possèdent au moins un animal de petit élevage. Les animaux sont principalement des poules, des canards, des dindons (figure 5) et des cochons, les poules étant les animaux les plus fréquemment élevés. La vente des produits du petit élevage $n^{\prime}$ est pas rare : $73 \%$ des éleveurs vendent au moins un animal par année, et $23 \%$ des ménages éleveurs vendent des œufs. Les animaux sont principalement vendus en période de soudure ou lorsque le ménage juge en posséder beaucoup. 
Tableau 3. Analyse financière du grand élevage dans deux villages du Menabe Central $(n=80)$ se focalisant sur la production bovine et caprine.

\begin{tabular}{|lrcrcr|}
\hline & \multicolumn{2}{c}{ Ampataka } & & \multicolumn{2}{c|}{ Ankoraobato } \\
\cline { 2 - 3 } \cline { 5 - 6 } & Montant (US\$) & Pourcentage & & Montant (US\$) & Pourcentage \\
\hline Coûts moyens annuels & & & & & \\
Entrées d'animaux & -3919 & 25 & & -3592 & 15 \\
Traitements vétérinaires & -19 & 0 & & -101 & 0 \\
Gardiennage & -10996 & 70 & & -19096 & 80 \\
Suppléments alimentaires & -751 & 5 & & -1217 & 5 \\
Total & -15685 & 100 & & -24006 & 100 \\
Revenus moyens annuels & 7238 & 55 & & 14124 & \\
Sorties d'animaux & 5847 & 45 & & 16198 & 53 \\
Ventes de lait & 13085 & 100 & & 30322 & 100 \\
Total & -2600 & & & 6316 & \\
Somme des coûts et des revenus & & & & & \\
\hline
\end{tabular}

1 US\$ $=1878$ MGA

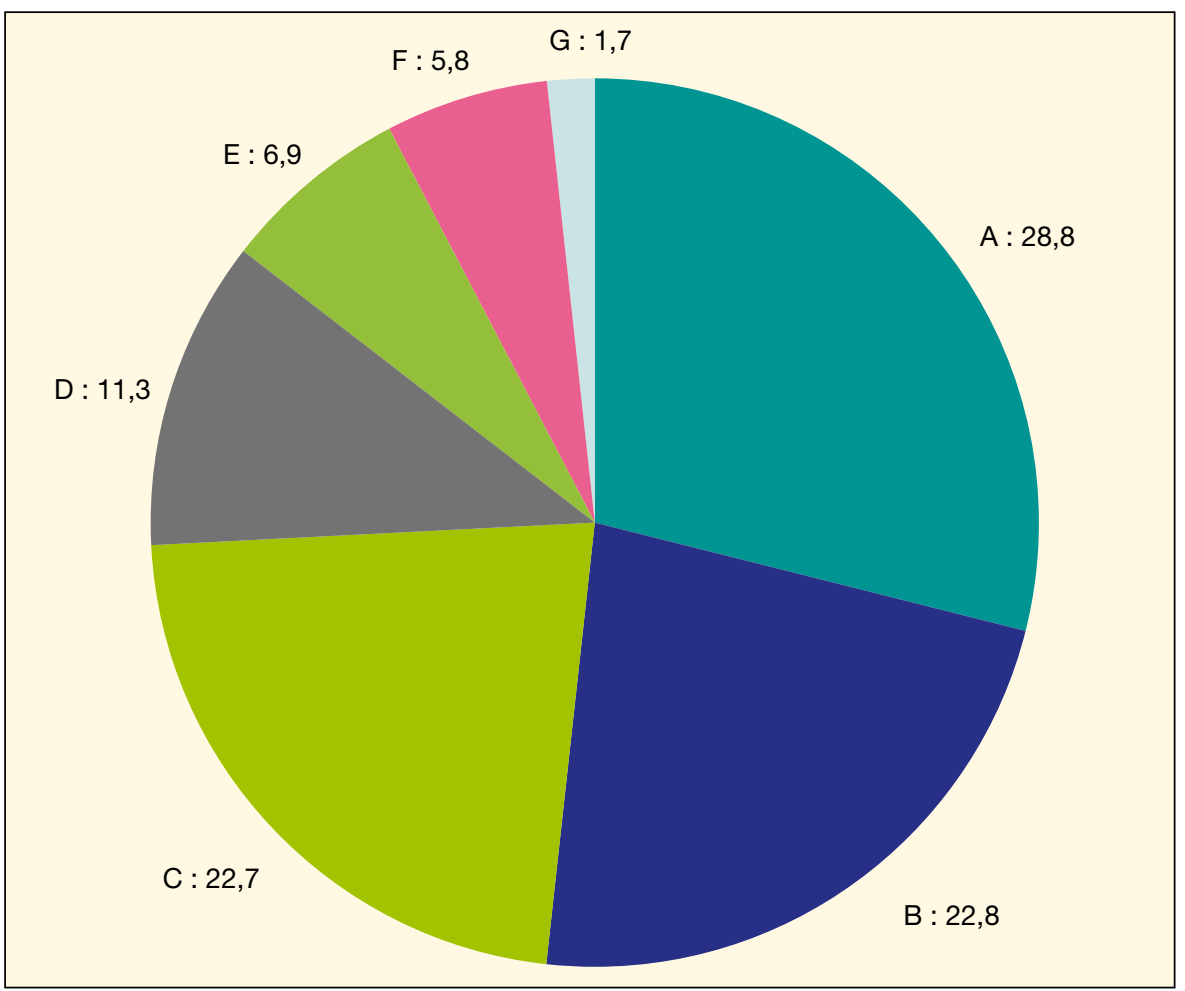

Figure 4. Préférences locales vis-à-vis de nouvelles techniques concernant le grand élevage. (scoring: pourcentages, $n=12$ groupes de 10 personnes). Les catégories sont: A) fixer les lieux de pâture ; B) parquer les zébus (fumier) ; C) arrêter les feux ; D) cultiver du foin ; E) améliorer les pâturages ; F) faire une rotation des lieux de pâture; $G$ ) autre.

Généralement, ces ventes se font pour des urgences (maladies, pénurie de nourriture, besoin d'argent immédiat, etc.) ou dans un but particulier (scolarisation des enfants, pétrole, café, vêtements, etc.). Globalement, l'écoulement commercial des produits du petit élevage ne pose pas de problème. En effet, $100 \%$ des éleveurs vendeurs de cochons affirment qu'il est
L'exercice de scoring aboutit au constat que le rôle du petit élevage est avant tout économique. En effet, les produits du petit élevage sont statistiquement $(U: p<0,01)$ majoritairement considérés comme une caisse d'épargne pour le ménage (figure 6).

Le principal problème du petit élevage semble être les maladies épidémiques des poules, car lors des enquêtes qualitatives, c'est l'unique problème cité dans tous les villages enquêtés. $D^{\prime}$ après les informations obtenues lors de ces enquêtes, des vaccins contre les maladies aviaires existent, mais ils coûtent chers et certains éleveurs n'y ont pas accès car les vétérinaires ne visitent pas tous les villages. De plus, lors des rares passages de vétérinaires dans les villages, les éleveurs n'ont pas forcément l'argent liquide pour acheter les médicaments adéquats.

Les enquêtes fournissent également des données qualitatives sur l'impact du petit élevage sur la biodiversité. Le petit élevage ne crée pas de problèmes écologiques directs. En effet, les poules entrent parfois en forêt pour se nourrir, ou des éleveurs collectent occasionnellement des termites pour les poussins, mais ces impacts sont négligeables car irréguliers. Certains éleveurs surveillent même leurs poules afin de ne pas les laisser aller en forêt, car il y a trop d'animaux sauvages. Les autres animaux du petit élevage $n$ 'entrent que rarement en forêt.

Le scoring sur les attentes locales vis-à-vis de la filière "petit élevage » montre que les trois techniques qui ont statistiquement $(U: p<0,01)$ le plus intéressé les éleveurs sont un passage fréquent et régulier de vétérinaires dans les villages, I'introduction de nouvelles espèces animales et des formations sur le petit élevage (figure 7 ).

\section{Discussion}

\section{Les pâturages}

Au Menabe central, les problèmes de feu et de surpâturage sont principalement liés au mode de gestion locale des pâturages. Suite à l'action prolongée et itérative des feux, couplée au lessivage du sol par différents facteurs climatiques (vents, eaux de ruissellement, etc. [1 11$])$, des espèces pyrophytes et envahissantes, à faible production fourragère (telles Brachiaria nana et Panicum voeltzkowii) ont remplacé peu à peu des espèces plus productives (Heteropogon contortus, Hyparrhenia rufa, Cynodon dactylon). Le manque de régénération naturelle de certaines graminées s'explique également par le fait que, après le passage du feu, les jeunes pousses sont broutées ou piétinées pendant la période d'épiaison. 


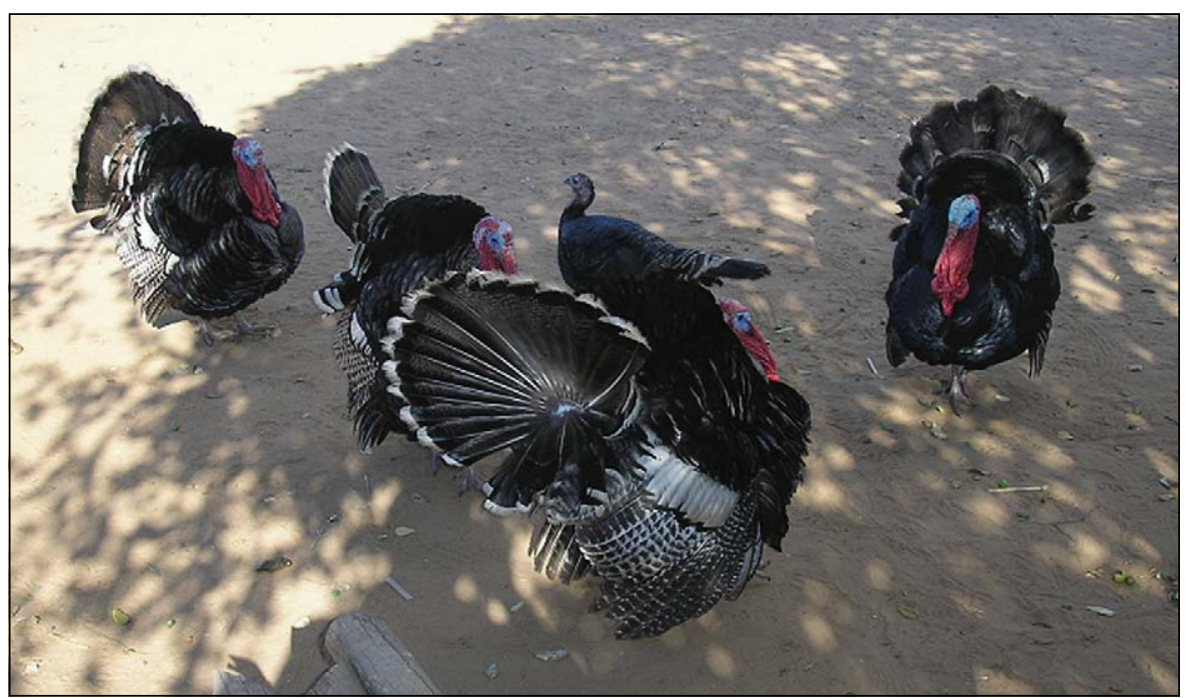

Figure 5. Des dindons sont élevés dans certains villages du Menabe central (cliché : L. H. Andriambelo).

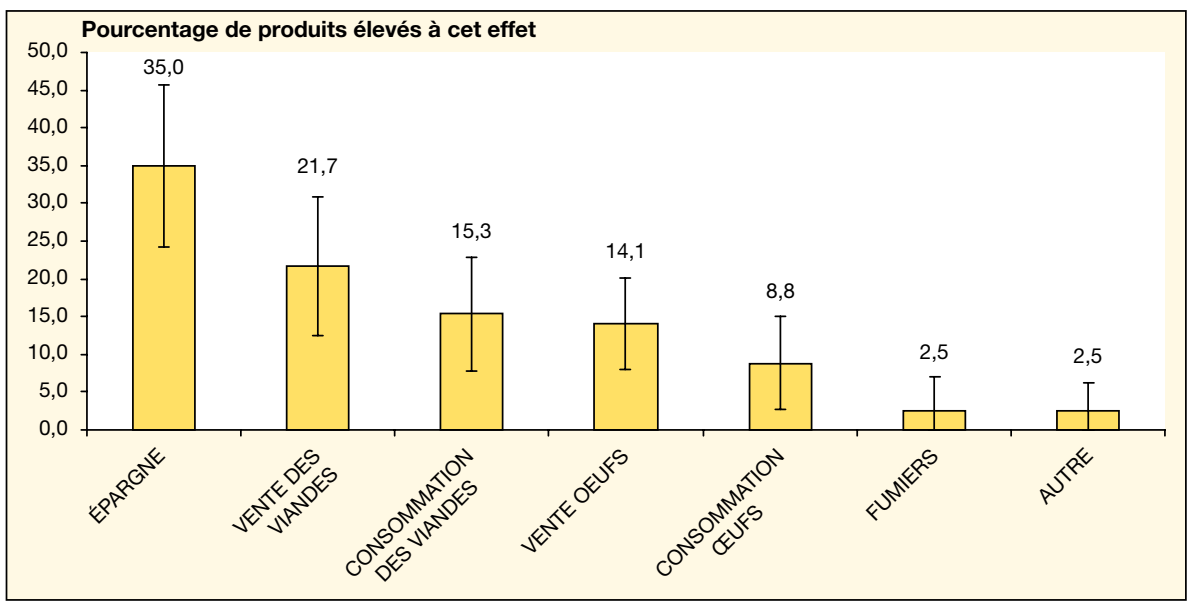

Figure 6. Importance relative des rôles des produits du petit élevage pour les ménages ruraux du Menabe central. (scoring: moyennes et écarts types, $n=12$ groupes de 10 personnes).

L'épargne représente les animaux qui sont utilisés comme caisse d'épargne, et qui sont vendus pour des besoins urgents.

Vingt-neuf pour cent de la surface pâturée montrent des traits de surpâturage en saison sèche. Les troupeaux pâturent le plus souvent à proximité des points d'eaux et dans les savanes à dominance d'Heteropogon contortus et à présence d'Hyparrhenia rufa et Cynodon dactylon. En effet, ces trois espèces sont plus appréciées et plus appétées par les bovins que les espèces pyrophytes. Malgré le surpâturage, les éleveurs pensent que les lieux de pâture doivent rester fixes, autrement dit que les itinéraires de pâture ne doivent pas changer ${ }^{1}$.

\footnotetext{
${ }^{1}$ Le choix des éleveurs pour des lieux de pâture fixes semble en contradiction avec la présence de surpâturage. Néanmoins, si les éleveurs ont insisté sur le tait de garder les mêmes lieux de pâture, c'est pour ne pas déstabiliser les zébus.
}

Influence de la commercialisation du lait sur la rentabilité du système " grand élevage»

Les éleveurs d'Ankoraobato d'ethnies immigrantes Antesaka et Antandroy exploitent leurs troupeaux d'une manière économique raisonnable, par rapport à l'ethnie indigène Sakalava d'Ampataka. Cela confirme que les éleveurs Sakalava attribuent une valeur plus culturelle que financière à leurs troupeaux [3]. Les rôles et objectifs de l'élevage sont donc déterminants pour la rentabilité économique de l'activité. En comparant les gestions des deux terroirs, on remarque que la production de lait contribue à $53 \%$ des revenus à Ankoraobato et à $45 \%$ à Ampataka. Alors qu'elle touche un petit nombre d'éleveurs et que la production laitière par vache est faible, la commercialisation du lait est un facteur important favorisant la rentabilité économique du système " grand élevage », comme cela a été constaté ailleurs en Afrique [12]. Au Menabe central, l'intensification du système pastoral passe donc par une augmentation des traites et des ventes de lait, car la filière laitière représente un marché rural prometteur [13]. Toutefois, la réussite de cette restructuration nécessite une sensibilisation et une responsabilisation de la communauté locale, tout comme une assistance technique (difficulté du stockage, stabilisation des prix) et des formations renforçant les capacités locales [14].

\section{Le rôle socio-économique du grand et du petit élevage}

La commercialisation du lait jove un rôle prépondérant dans la rentabilité du système "grand élevage ", mais paradoxalement, les éleveurs ne traient pas les chèvres, et uniquement $7 \%$ des éleveurs de bovins vendent parfois du lait. Cela laisse penser que même dans un système économiquement rentable, le rôle du système "grand élevage " n'est pas intensément lucratif. Par contre, $73 \%$ des éleveurs de petits animaux vendent annuellement des bêtes, et $23 \%$ vendent des œufs. Ceci met en évidence le fait qu'il est dans la coutume des éleveurs du Menabe central de vendre des produits du petit élevage et de garder ceux du grand élevage. En outre, les produits du petit élevage se vendent facilement au Menabe central, et les ventes de volailles sont généralement gérées par les femmes [15], ce qui signifie que leurs recettes sont directement intégrées dans les fonds du ménage. Intensifier le système "petit élevage » pourrait donc être bénéfique au développement rural du Menabe central, à condition d'orienter les efforts vers un appui à l'amélioration 


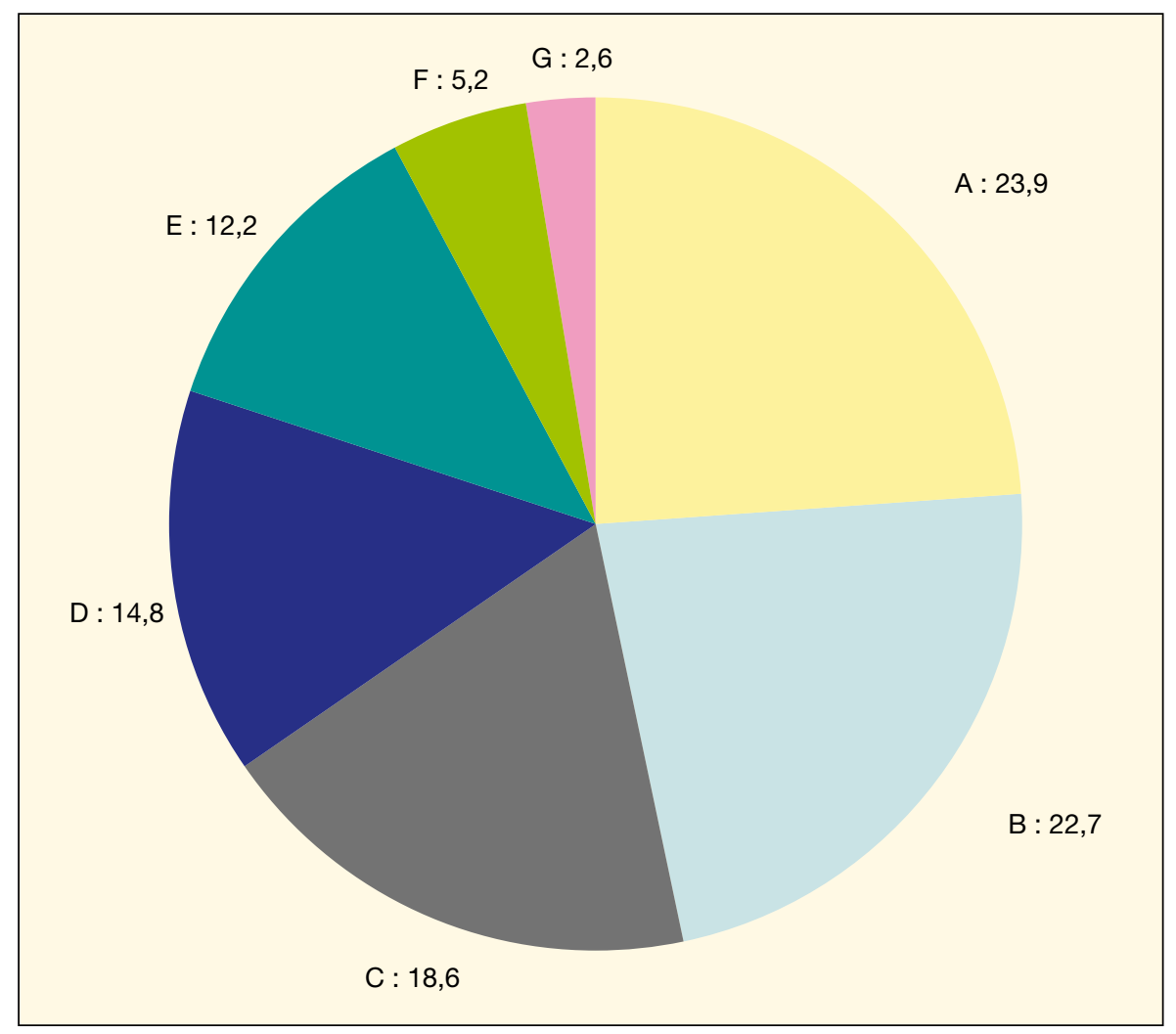

Figure 7. Préférences locales en vue de l'amélioration de la filière « petit élevage ».

(scoring: pourcentages, $n=12$ groupes de 10 personnes). Les catégories sont: A) augmentation du nombre de passages de vétérinaires au village ; B) introduction de nouvelles espèces; C) formation ; D) vaccination de groupes d'animaux; E) augmentation des acheteurs en gros; F) organisation des transports en groupe pour les ventes; G) autre.

sanitaire des poules en proposant, par exemple, des passages plus fréquents de vétérinaires dans les villages ou des solutions de microcrédits qui faciliteraient l'accès aux médicaments pour les animaux. Toutefois, la réussite de cette intensification nécessite une assistance technique et des formations rurales afin de renforcer les pratiques locales.

\section{Conclusion}

Au Menabe central, une réorganisation spatiale et temporelle des terrains pâturés (par exemple, une mise au repos alternative de certaines parcelles pâturées sur un itinéraire) diminuerait l'impact du surpâturage et du feu sur la biodiversité; cette restructuration nécessite tout $d^{\prime}$ 'abord une sécurisation foncière des lieux de pâture. Économiquement, des traites et des ventes de lait plus fréquentes développeraient le

\section{Références}

1. Scales I. Understanding the past reframing the present : forest loss and landscape change in western Madagascar. Norwich: Society, Natural Resources and Development in Madagascar, Recent Contributions by the Research Community, 2007.

2. CNRE; Orstom. Le développement de l'élevage dans le sud-ouest de Madagascar. Morondava (Madagascar) : CNRE, Orstom, sd.

3. Région du Menabe, CRD Menabe. Région du Menabe, Programme Régional de Développement. Antananarivo (Madagascar) : République de Madagascar.

4. Kaimowitz D, Sheil D. Conserving what and for whom? why conservation should help meet basic human needs in the tropics. Biotropica 2007; 39 : 567-74.

5. PNAE. http://www.pnae.mg/ie/tbe/itasy/ socioeco/donnees/pauvrete.htm, 2008 [2 décembre 2008].

6. Braun-Blanquet J. Pflanzensoziologie. Grundzüge der Vegetationskunde. Wien; New-York: Springer, 1964

7. Gounot M. Méthode d'études quantitatives de la végétation. Paris : Masson, 1969.

8. Boudet $G$. Problèmes posés par l'estimation de la capacité de charge d'un pâturage naturel tropical. Collection de Référence Orstom 1975 ; (8655): 265-7.

9. MAEP ; UPDR ; CIREL. Monographie de la région du Menabe. Antananarivo (Madagascar) : MAEP, 2003.

10. Sheil D, Liswanti N. Scoring the importance of tropical forest landscapes with local people : patterns and insights. Environmental Management 2006 ; 38 : 126-36.

potentiel du système "grand élevage », et l'amélioration des conditions sanitaires (par des vaccins, des visites vétérinaires plus fréquentes, des possibilités de microcrédits, etc.) - en particulier pour les volailles - diminuerait les pertes; I'augmentation des traites de zébus et de chèvres et l'accroissement du nombre d'animaux du petit élevage ne présentent pas d'impacts négatifs sur les forêts sèches.

Bien que cette étude soit participative, la réussite de tout changement nécessite l'acceptation des recommandations par les populations locales. À cet égard, l'avis des éleveurs sur les recommandations doit être analysé. De plus, une assistance technique et des formations rurales à même de renforcer les pratiques locales sont primordiales. L'influence d'autres activités villageoises, telle l'agriculture, doit également être prise en compte dans une perspective $d^{\prime}$ approche globale et de durabilité de l'utilisation traditionnelle des espaces forestiers.
11. Bellefontaine R, Gaston A, Petrucci Y. Aménagement des forêts naturelles des zones tropicales sèches. Rome : FAO, 1997.

12. Itty P, Rowlands GJ, Traub D, Hecker P, Coulibaly L, D'leteren $G$. Étude économique de la production bovine villageoise dans une région du nord de la Côte d'Ivoire infestée par les glossines. Revue d'Elevage et de Médecine Vétérinaire des Pays Tropicaux 1994 ; 47 : 333-43.

13. Dirac Ramohavelo C. Stratégies villageoises dans la gestion des paysages forestiers, Menabe Central Madagascar. Lausanne : Ecole Polytechnique Fédérale de Lausanne, 2009.

14. FERT/CNEAP. Collèges agricoles à Madagascar. Partenariat FERT/CNEAP, 2006. hittp:// cneap.scolanet.org/BibliRessources/PagesSystem /ViewNodeFile. ashx?idnode $=3433$

15. Allab C. Étude des budgets des ménages du Menabe Central. Propositions méthodologiques et éléments de compréhension des stratégies économiques des familles paysannes. Morondava (Madagascar) : Programme Menabe, 1999. 